\title{
MicroRNA And Circular RNA Expression In Affected Skin Of Patients With Postherpetic Neuralgia
}

This article was published in the following Dove Press journal:

Journal of Pain Research

\author{
Song $\mathrm{Cao}^{1,2}$ \\ Dexin Zhang' \\ Jie Yuan ${ }^{1,2}$ \\ Chengxi Liu ${ }^{2}$ \\ Wenjing Zhou ${ }^{2}$ \\ Lin Zhang ${ }^{2}$ \\ Shouyang $\mathrm{Yu}^{2}$ \\ Bangyong Qin' \\ Ying $\mathrm{Li}^{\mathrm{I}}$ \\ Wenwen Deng ${ }^{3}$ \\ 'Department of Pain Medicine, Affiliated \\ Hospital of Zunyi Medical University, \\ Zunyi 563000, People's Republic of \\ China; ${ }^{2}$ Guizhou Key Laboratory of \\ Anesthesia and Organ Protection, Zunyi \\ Medical University, Zunyi 563003, \\ People's Republic of China; ${ }^{3}$ Department \\ of Cardiology, Affiliated Hospital of Zunyi \\ Medical University, Zunyi 563000, \\ People's Republic of China
}

Correspondence: Song Cao

Department of Pain Medicine, Affiliated

Hospital of Zunyi Medical University,

Zunyi 563000, People's Republic of China

Tel +86I82I2I70434

Email caosong432I@I63.com

Wenwen Deng

Department of Cardiology, Affiliated

Hospital of Zunyi Medical University,

Zunyi 563000, People's Republic of China

Tel +86I5085064354

Email912395627@qq.com

\begin{abstract}
Mechanisms of postherpetic neuralgia (PHN) are still not clear. Transcripts such as microRNA (miRNA) and circular RNA (circRNA) in the affected skin may take part in the initiation and development of this neuropathic pain; however, their expression profiles in skins of PHN patients have not been reported. The PHN affected skin and the mirror skin were collected and subjected to miRNA and circRNA microarray, and expression profiles were comparatively analyzed. There were 317 differently expressed miRNAs in PHN affected skin compared with mirror skin (fold change $\geq 2.0$ ), and 13 of them showed fold change $>10$ in the PHN skin. Only one circRNA, hsa_circRNA_405463 showed fold change $>2$ in PHN skin, however, 31 circRNAs with fold change $\geq 1.5$. To evaluate functions of differential miRNAs, their target mRNAs were predicted and bioinformatics analyses including gene ontology, Kyoto Encyclopedia of Genes and Genomes pathway were conducted. Target mRNAs significantly $(\mathrm{P}<0.05)$ enriched in 85 pathways, such as FoxO, AMPK, MAPK and pathway. These data reported for the first time that miRNA and circRNA differentially expressed in the PHN skin and these transcripts with abnormal expression could be potential targets to treat PHN.
\end{abstract}

Keywords: neuropathic pain, postherpetic neuralgia, microRNA, circular RNA

\section{Introduction}

Postherpetic neuralgia (PHN) is a typical neuropathic pain, which lasts more than 1 month, ${ }^{1}$ and profoundly affects the quality of life. ${ }^{2}$ The mechanisms of PHN are complicated, including the peripheral pathology ${ }^{3-5}$ and plastic changes in the central nervous system. ${ }^{4-8}$ Elucidating molecular changes during neuropathic pain is fundamental for the development of mechanism-oriented treatments. ${ }^{9}$ Understanding the molecular changes in PHN skin may help develop skin-targeting external medications or treatments.

MicroRNAs (miRNAs) are small non-coding molecules, which post-transcriptionally modulate pathological processes including neuropathic pain. ${ }^{10,11}$ Existing studies have shown that miRNAs are involved in the development of neuropathic pain and are expected to become clinical biomarkers and intervention targets. ${ }^{12,13}$ miRNA expression differences were detected in serum between two kinds of patients: herpes zoster and PHN. ${ }^{14}$ In patients with peripheral neuropathy, abnormal expression of miR-21-5p, miR-146a, miR-132-3p and miR-155 were reported in the skin, blood leukocytes and sural nerves. ${ }^{15,16}$ Patients with fibromyalgia syndrome showed abnormal expression of miR-let-7d in skin and leukocytes, which may be associated with lesions in peripheral skin neurofibromas. ${ }^{17}$ 
Circular RNAs (circRNAs) are non-coding RNAs which interact with miRNAs. ${ }^{18}$ circRNAs can act as miRNA sponges $^{19-21}$ and regulate gene expression through a circRNA-miRNA-mRNA pattern. ${ }^{22,23}$ To date, there are few reports on the expression change and mechanism of circRNA in pain situation. ${ }^{24-26} \mathrm{We}$ previously found that circRNA expression profile changed in the dorsal spinal cord of CCI rats. ${ }^{27}$ It is reported that intrathecally silence circHIPK3 with shRNA alleviated neuropathic pain in diabetic rats. ${ }^{24}$

It seems feasible and effective to interfere with the expression of miRNAs in skin. Animal studies suggested that interfering with the expression of miRNAs in the skin may affect the prognosis of diseases. Skin is an important lesion site and potential drug delivery location ${ }^{3}$ in PHN; however, the expression profiles of miRNA and circRNA in PHN skin have not been reported. In this study, the differential expression of miRNA and circRNA were detected with microarrays, and differential miRNAs' target mRNAs were subjected to gene ontology (GO) and Kyoto Encyclopedia of Genes and Genomes (KEGG) pathway analyses to evaluate their possible functions and mechanisms in PHN situation.

\section{Methods}

\section{Participants}

This study was conducted in accordance with the Declaration of Helsinki. The present study was approved by the Medical Ethics Committee of the Affiliated Hospital of Zunyi Medical University and registered at the Chinese Clinical Trial Registry (ChiCTR1800017821) and all patients provided written informed consent. Patients were recruited from the Department of Pain Medicine of the local hospital from September 2018 to March 2019. All PHN patients in this study were diagnosed by two or more chief physicians. Pain intensity was evaluated with an 11-point numeric rating scale (NRS-11, values from 0 to 10). All of the patients recruited claimed intense pain (NRS scores $\geq 5$ ) and pain durations were more than 1 month after the HZ healed. For easy sampling and to ensure that the skin locations were identical and comparable, only PHN lesion in skin of the thoracic segment was enrolled. Five PHN patients were enrolled in the microarray experiments and described in Table 1.

\section{Skin Collection}

For each patient, two circular skins on the back were sampled. Patients took a prone position, local anesthetics ( $1 \%$ lidocaine) was injected at the lesion site and the mirror site (symmetrical site of the opposite side) before the skin was taken with biopsy punchers (with $5 \mathrm{~mm}$ diameter and circular cross section). The bottom of the skin samples reaches the dermis, with a thickness of $\sim 2 \mathrm{~mm}$.

\section{RNA Isolation, Purification And Hybridization}

Total RNA from each sample was isolated and purified as we reported. ${ }^{28}$ miRNA labeling and array hybridization was according to Exiqon's manual. After stopping the labeling procedure, the $\mathrm{Hy} 3^{\text {TM}}$-labeled samples were hybridized on the miRCURYTM LNA Array (v.19.0, Exiqon, USA) according to array manual. Then, the slides were scanned using the Axon GenePix 4000B microarray scanner (Axon Instruments, Foster City, USA).

For circRNA microarray, the RNA preparation and microarray hybridization were performed as we reported ${ }^{27}$ based on Arraystar's standard protocols. ${ }^{29}$ Total RNA from each sample was treated with Rnase R (Epicentre Inc.) to remove linear RNAs and to enrich circRNA. Then, the enriched circRNAs were amplified and transcribed into fluorescent cRNA using a random priming method (Arraystar Super RNA Labeling Kit; Arraystar, USA). The labeled cRNAs were hybridized onto the Arraystar Human circRNA Array (v.2.0, 8×15K, Arraystar). At last, the arrays were scanned by the Agilent Scanner G2505C (Agilent, USA).

\section{Microarray Data Analysis}

For miRNAs, scanned images were imported into GenePix Pro 6.0 software (Axon) for grid alignment and data

Table I Demographic And Clinical Variables Of Five Patients In Microarray Experiments

\begin{tabular}{|l|l|l|l|l|l|}
\hline No. & Age (Year) & Gender & Location Of Lesion & Pain Duration (Month) & NRS Score \\
\hline I & 65 & M & Right T2-4 & 24.0 & 7 \\
2 & 74 & M & Right T5-7 & 1.0 & 9 \\
3 & 64 & M & Left T6-8 & 1.0 & 6 \\
4 & 77 & F & Right T4-6 & 1.0 & 7 \\
5 & 63 & F & Left T3-5 & 12.0 & 8 \\
\hline
\end{tabular}

Abbreviations: M, male; F, female; T, thoracic; NRS, numerical rating scale. 
extraction. Replicated miRNAs were averaged and miRNAs that intensities $\geq 30$ in all samples were chosen for calculating normalization factor. Expressed data were normalized using the median normalization. For circRNAs, quantile normalization of raw data and subsequent data processing were performed using the R software limma package. After quantile normalization of the raw data, low-intensity filtering was performed, and the circRNAs that at least 5 of 10 samples have flags in "P" or "M" ("All Targets Value") were retained for further analyses. After normalization, significant differentially expressed miRNAs and circRNAs between two groups were identified through fold change and P-value. MiRNAs and circRNAs with fold changes $\geq 2$ and P-value $\leq 0.05$ were selected as differentially expressed genes.
Hierarchical clustering was used to display the distinguishable expression pattern between PHN and control samples.

\section{Real-Time Quantitative PCR Validation}

Five differential miRNAs were randomly selected and subjected to real-time quantitative PCR (RT-qPCR). RNAiso Plus (Takara, Japan) was used to isolate total RNA from skins according to manufacturer's instructions. Total RNA was prepared as standard template for reverse transcription with the Mir-X miRNA First-Strand Synthesis Kit (Clontech Laboratories, Japan). The miRNA-specific 5' primers were as follows: hsa-miR3664-3p, 5'-CGTCTCAGGAGTAAAGACAGAGTT-3'; hsa-miR-4714-3p, 5'-CCAACCTAGGTGGTCAGAGTT-
A
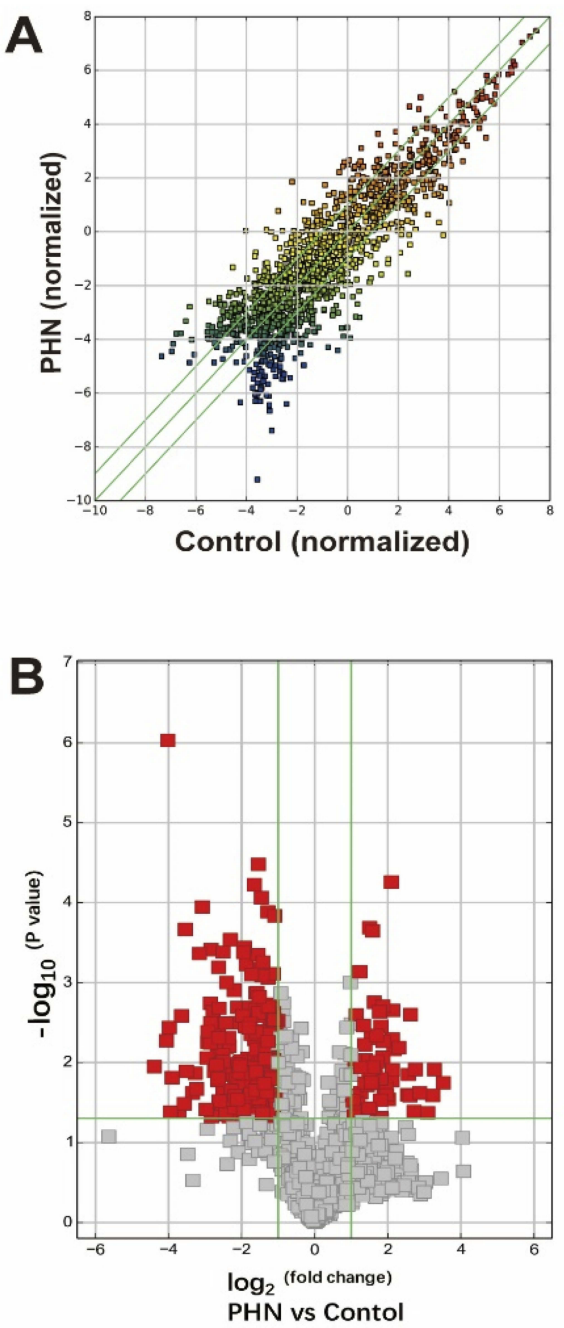
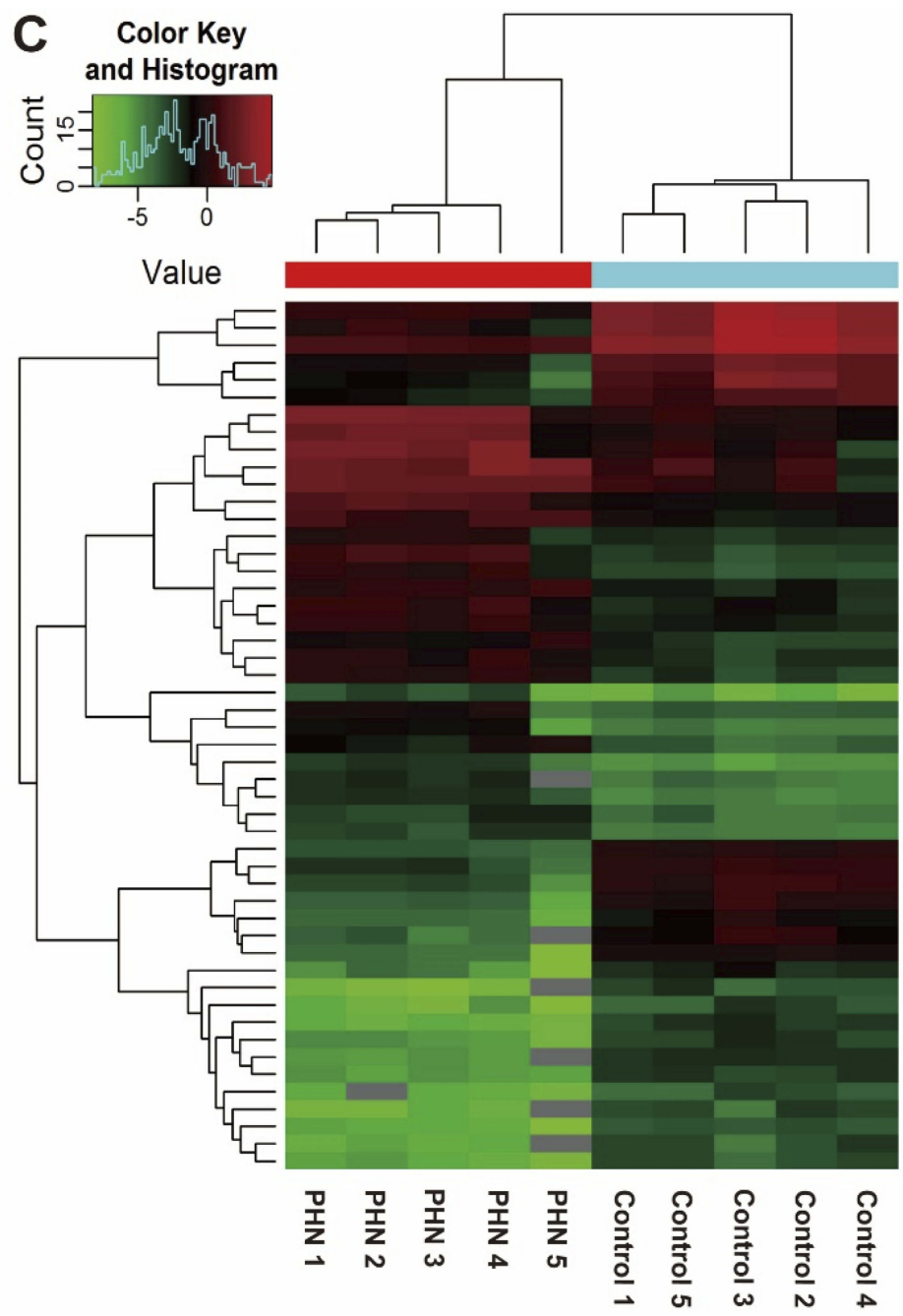

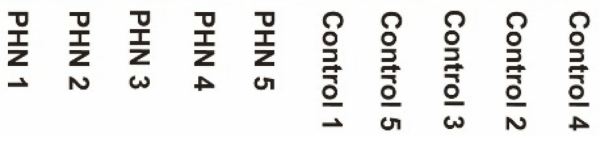

hsa-miR-4714-3p hsa-miR-4714-3p hsa-miR-410-5p hsa-miR-604
hsa-miR-5579-3p hsv1-miR-H4-5p hsa-miR-1258 hsa-miR-29b-1-5p
hsa-miR-382-5p hsa-miR-382-5p hsa-miR-4657 hsa-miR-548e-3p hsa-miR-605-5p
hsa-miR-3160-3p hsa-miR-4491 hsa-miR-181a-2-3p hsa-miR-1265 hsa-miR-4307 hsa-miR-885-3p
hsa-miR-4754 hsa-miR-4506 hsa-miR-4721 hsa-miR-760 hsa-miR-495-3p hsa-miR-330-5p hsa-miR-4759 hsa-miR-381-5p hsa-miR-4458 hsa-miR-1282 hsa-miR-4680-3p hsa-miR-2682-5p hsa-miR-3139
hsa-miR-4692 hsa-miR-3678-3p hsa-miR-3194-5p hsa-miR-302a-5p hsa-miR-3678-5p hsa-miR-5008-5p hsa-miR-649-5p hsa-miR-503-3p hsa-miR-3187-3p
hsa-miR-3170 hsa-miR-3975 hsa-miR-455-3p

Figure I miRNA expression comparison between the PHN skin and normal skin. (A) Scatter plot shows the miRNA expression differences between two groups. The miRNAs above the top green line and below the bottom green line indicated differential miRNAs (fold change $\geq 2.0$ or $\leq-2.0$, respectively). (B) The volcano plot shows the distribution of differential miRNAs (indicated with red blocks). Horizontal green line indicated the threshold of $\mathrm{P}$-value, above which indicates $\mathrm{P}$ - values $<0.05$; left and right vertical green line indicated fold change threshold of -2.0 and 2.0, respectively, and between which indicates genes with fold changes $<2.0$. (C) Hierarchical clustering of top 25 up- and down-regulated miRNAs between groups. 
3'; hsa-miR-16-5p, 5'-CCGTAGCAGCACGTAAATATT GGCG-3'; hsa-miR-20a-5p, 5'-CGGCTAAAGTGCTTAT AGTGCAGGTAG-3'; hsa-miR-505-5p, 5'-ACGGGAGC CAGGAAGTATTGATGT-3'; hsa-let-7a-5p, 5'-CGGCGT GAGGTAGTAGGTTGTATAGTT-3'. RT-qPCR was performed on a CFX Connect Real-Time system (Bio-Rad, USA) in a $25 \mu \mathrm{L}$ tube containing $12.5 \mu \mathrm{L}$ of TB Green ${ }^{\mathrm{TM}}$ Premix Ex Taq ${ }^{\mathrm{TM}}$ II (Takara), and $0.5 \mu \mathrm{L}$ of mRQ 3' Primer according to manufacturer's instructions. U6 was used as an endogenous reference. The $2^{-\Delta \Delta \mathrm{Ct}}$ method was used to calculate the relative expression of miRNAs.

\section{MiRNA Prediction}

Target mRNAs of the top 100 differential miRNAs were predicted with Arraystar's home-made miRNA target prediction software based on the TargetScan (v.7.1, http://www. targetscan.org) ${ }^{30}$ and miRDB (v.5.0, http://mirdb.org/) prediction algorithm. Only the mRNAs predicted by two databases were included in the GO and pathway analyses.

\section{Bioinformatics Analysis}

GO and pathway analyses were performed to explore the function of differential miRNAs. The mRNAs of top 100 differential miRNAs were subjected to GO annotation in terms of Biological Processes, Cellular Components and
Molecular Functions. Pathways defined by KEGG, Biocarta and Reactome (http://www.genome.jp/kegg/) were identified by DAVID (https://david.ncifcrf.gov/).

\section{Statistical Analysis}

The results were reported as mean \pm SD. Statistically significant differences of miRNA expression between PHN and control group were evaluated with paired $t$-tests with GraphPad Prism (v.7.0, GraphPad Software, USA). $P<0.05$ was considered to be statistically significant.

\section{Results}

\section{miRNA Profiles}

Ten samples from five PHN patients were subjected to miRNA microarray (Figure 1). The miRCURYTM LNA Array (v7.0, Exiqon) detected 2080 human miRNAs (Supplementary material 1). Overall, 317 of them were calculated as differential miRNAs (fold change $\geq 2.0, \mathrm{P}<0.05$ ), 67 were significantly up-regulated, and the other 250 were down-regulated in PHN group (Supplementary material 2). The miRNA, hsa-miR-4772-5p, down-regulated 21 times in PHN skin. Thirteen of these differentially expressed miRNAs showed expression fold change $>10$. The top 10 up- and down-regulated miRNAs are listed in Table 2.

Table 2 Differential miRNAs Between PHN Skin And Mirror Skin $(n=5)$

\begin{tabular}{|c|c|c|c|c|}
\hline MiRNA Name & Fold Change & Intensity (PHN) & Intensity (Control) & P-value \\
\hline \multicolumn{5}{|l|}{ Top 10 up-regulated in $\mathrm{PHN}$} \\
\hline hsa-miR-449I & 11.5 & 498 & 40 & 0.018 \\
\hline hsa-miR-502-5p & 9.6 & 304.7 & 29 & 0.012 \\
\hline hsa-miR-4528 & 9.3 & 116.7 & 11.5 & 0.026 \\
\hline hsa-miR-472I & 8.5 & 29.5 & 3.2 & 0.042 \\
\hline hsa-miR-760 & 7.3 & 171.9 & 21.8 & 0.024 \\
\hline hsa-miR-495-3p & 6.8 & 150.7 & 21 & 0.012 \\
\hline hsa-miR-382-5p & 6.6 & 1765.4 & 244.9 & 0.041 \\
\hline hsa-miR-4506 & 6.1 & 295.3 & 45 & 0.003 \\
\hline hsa-miR-I258 & 6.1 & 1839.7 & 278.9 & 0.026 \\
\hline hsa-miR-330-5p & 5.9 & 45.1 & 7.1 & 0.018 \\
\hline \multicolumn{5}{|l|}{ Top 10 down-regulated in PHN } \\
\hline hsa-miR-4772-5p & -21.0 & 1.2 & 33.6 & 0.011 \\
\hline hsa-miR-2682-5p & -16.7 & 18.8 & 293.4 & 0.005 \\
\hline hsa-miR-3678-3p & -16.2 & 13.7 & 205.7 & $9.3 \mathrm{E}-07$ \\
\hline hsa-miR-3678-5p & -15.8 & 3.4 & 51 & 0.004 \\
\hline hsa-miR-5579-3p & -15.5 & 97.9 & 1417.6 & 0.042 \\
\hline hsa-miR-3664-3p & -15.0 & 265.4 & 3719.7 & 0.015 \\
\hline hsa-miR-4692 & -13.2 & 15.4 & 241.6 & 0.041 \\
\hline hsa-miR-4680-3p & -12.5 & 31.9 & 373.8 & 0.003 \\
\hline hsa-miR-3|87-3p & -11.9 & 2.3 & 31.6 & 0.033 \\
\hline hsa-miR-518e-3p & -11.7 & 24.2 & 266.2 & 0.000 \\
\hline
\end{tabular}




\section{RT-qPCR Validation}

To validate the microarray data, RT-qPCR was employed to detect miRNA expression in PHN skin and normal mirror skin in another cohort of PHN patients $(n=12)$. Five miRNAs were randomly selected. Expression levels detected by the microarray and RT-qPCR are presented in
A

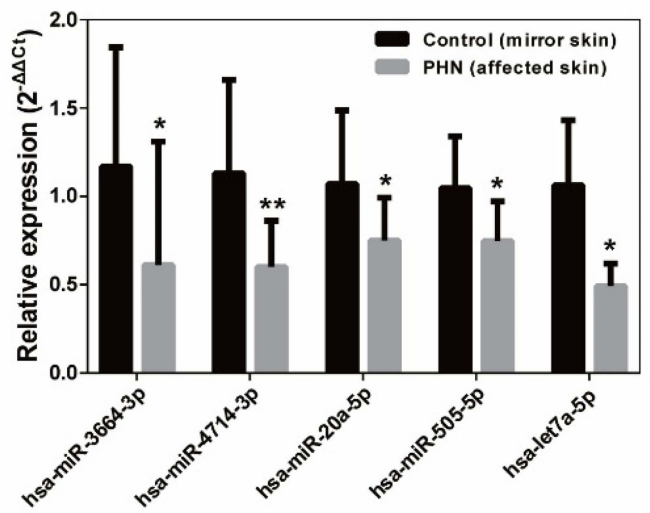

C

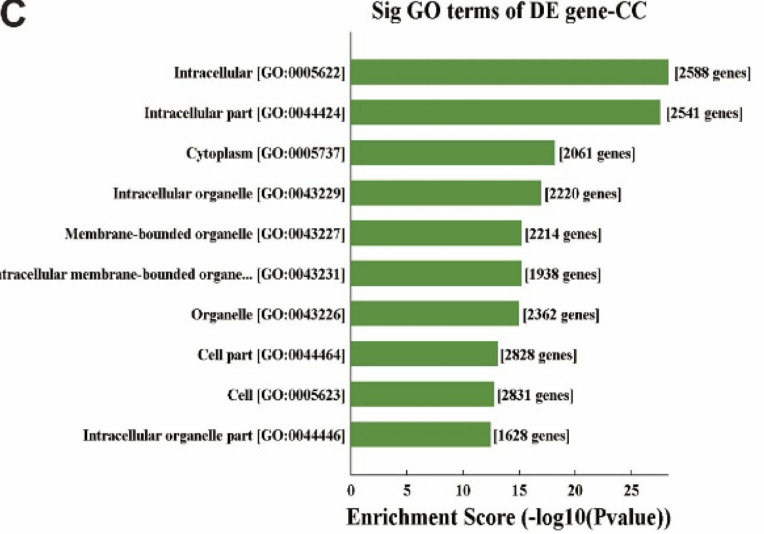

B

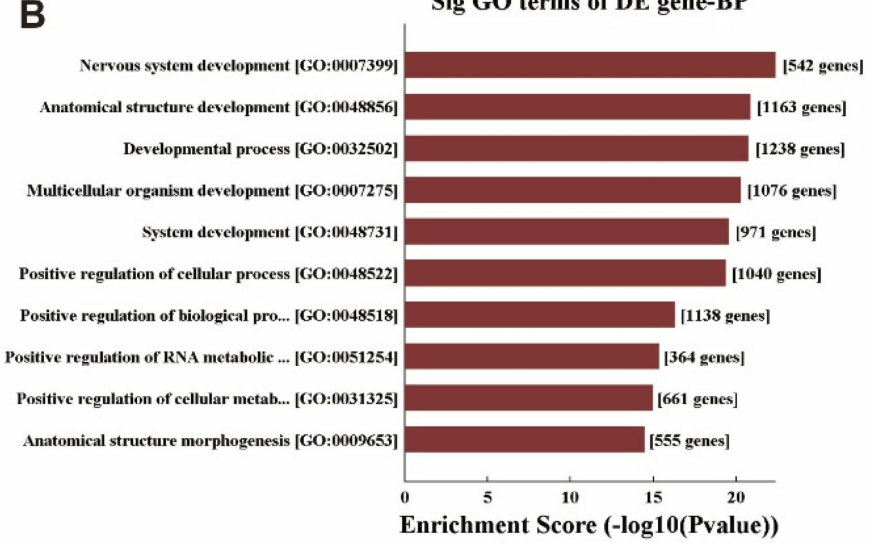

D

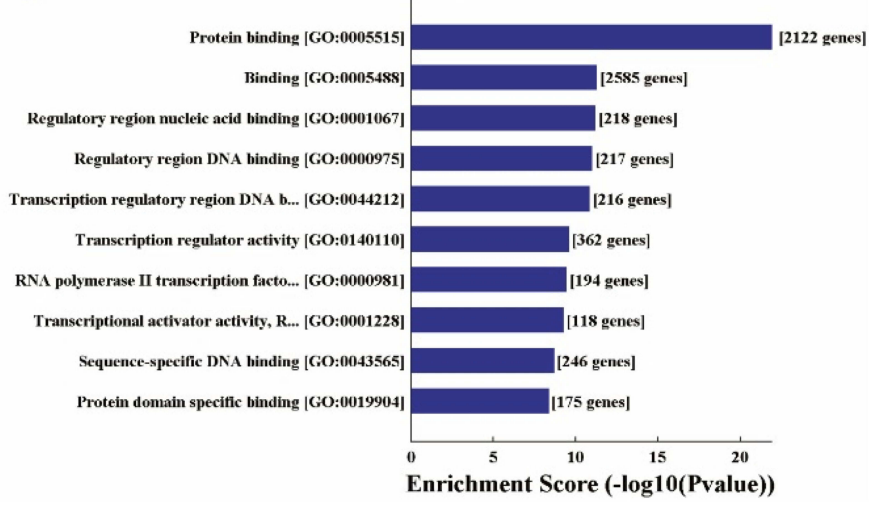

Figure 2 Validation of five differentially expressed miRNAs by RT-qPCR and gene ontology enrichment analysis. (A) Validation of five differentially expressed miRNAs by RTqPCR. Data were from 6 to 12 patients. Expression levels were normalized to U6 expression. Bars represent the mean $\pm S D$. Paired $t$-tests. $* P<0.05$; $* * P<0.0$ I compared with control group. (B-D) Enriched gene ontology (GO) terms correspond to the 4077 target mRNAs of the top 100 miRNAs. (B) Top 10 significantly (Sig) enriched biological processes (BP) terms. (C) Top 10 enriched cellular component (CC) terms. (D) Top 10 enriched molecular function (MF) terms.
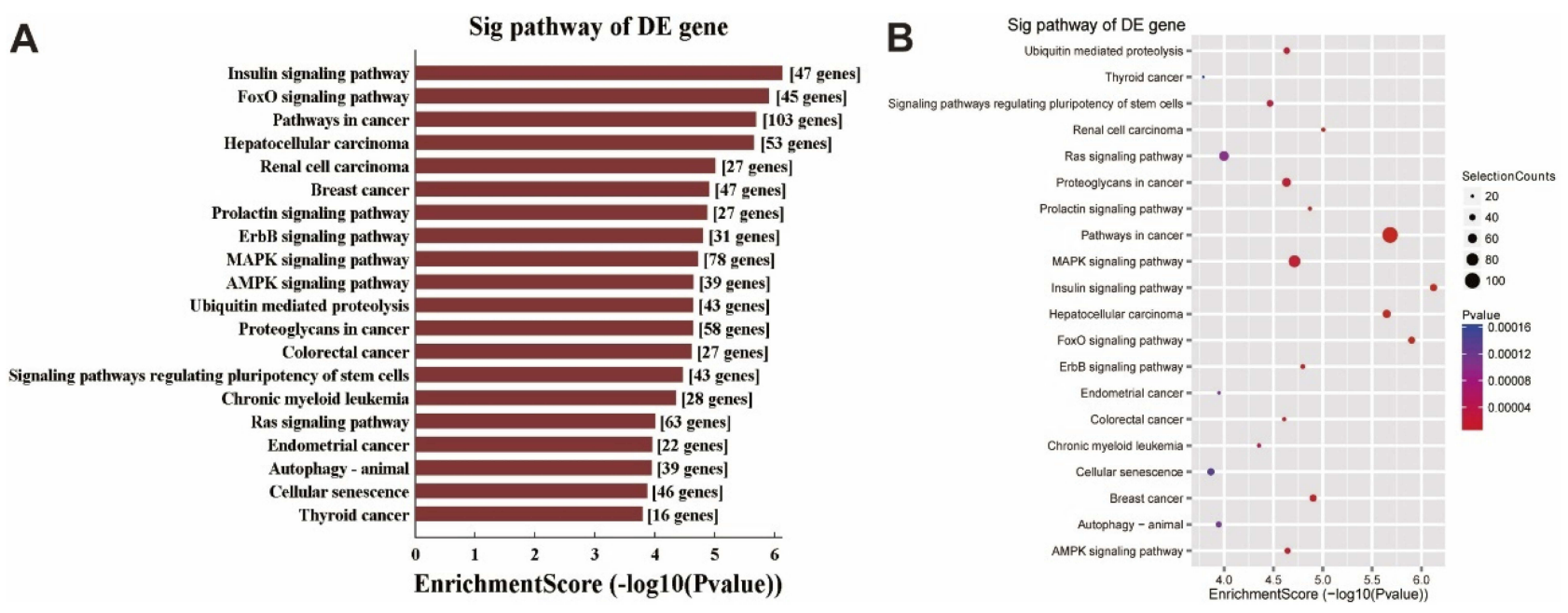

Figure 3 Top 20 KEGG pathways significantly enriched by target mRNAs of top 100 miRNAs. (A) Top 20 pathways significantly enriched $(P<0.05)$ by the 4077 target mRNAs of top 100 miRNAs. (B) The mRNA numbers enriched in the top 20 pathways. Selection counts refer to the count of mRNAs' entities directly associated with the listed pathway. 

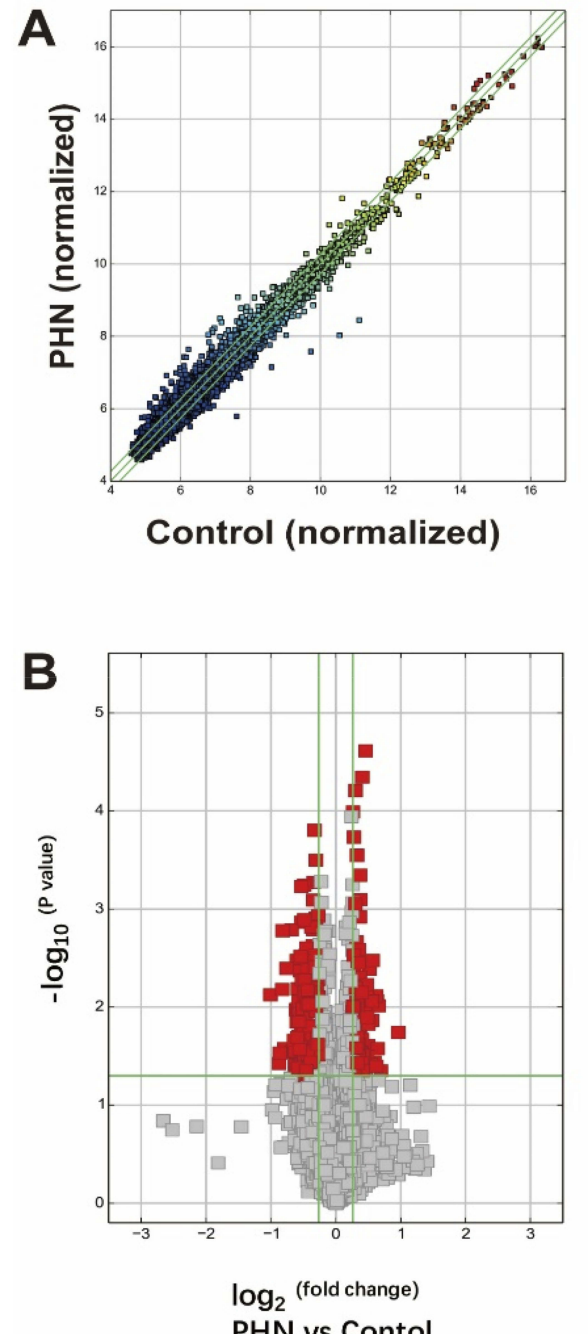
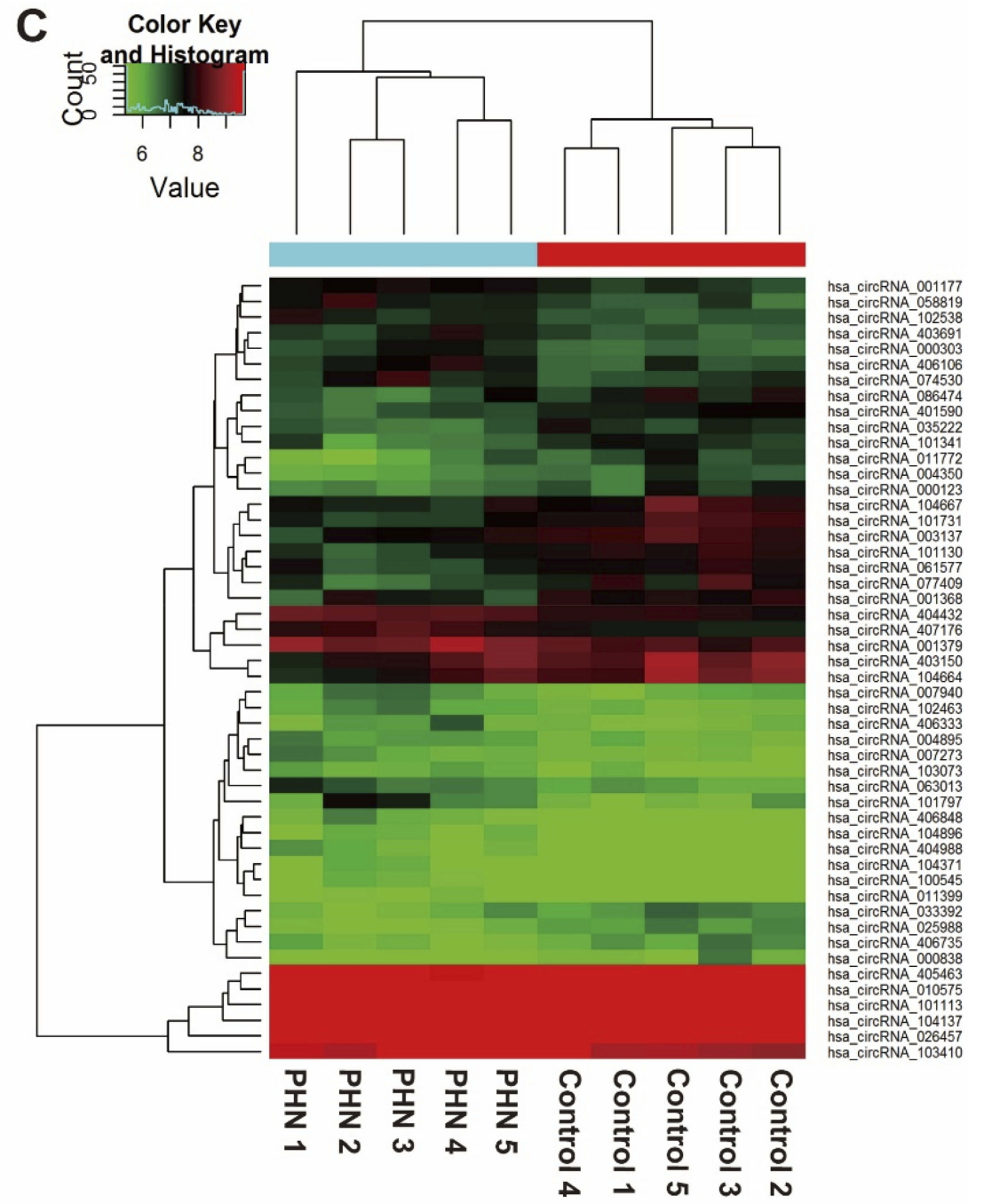

Figure 4 circRNA expression comparison between the PHN skin and normal skin. (A) Scatter plot shows the circRNA expression differences between two groups. The circRNAs above the top green line and below the bottom green line indicated differential circRNAs (fold change $\geq 1.2$ or $\leq-I .2$, respectively). (B) The volcano plot shows the distribution of differential circRNAs (indicated with red blocks). Horizontal green line indicated the threshold of $\mathrm{P}$-value, above which indicates $\mathrm{P}$ - values $<0.05$; left and right vertical green line indicated fold change threshold of -1.2 and I.2, respectively, and between which indicates genes with fold changes <I.2. (C) Hierarchical clustering of top 25 up- and down-regulated circRNAs between groups.

Figure 2A. The expression trends detected by the two methods were consistent, which suggested high reliability of the microarray results.

\section{Bioinformatics Analyses Of Target mRNA Of Differential miRNAs}

A total of 4077 target mRNAs of the top 100 miRNAs were predicted by miRDB and TargetScan. These mRNAs were subjected to GO enrichment and the top 20 significantly enriched $(P<0.05)$ GO terms are listed in Figure 2B-D. These mRNAs enriched in Biological Processes, Cellular Component and Molecular Function terms, such as Protein binding (GO: 0005515, with 542 genes) etc.
KEGG pathway analysis showed that the 4077 target mRNAs significantly $(P<0.05)$ enriched in 85 pathways (Supplementary material 3), and the top 20 pathways are listed in Figure 3. The FoxO (45 mRNAs), ErbB (31 mRNAs), MAPK (78 mRNAs) and AMPK (39 mRNAs) pathway were among the top 10 (Figure 3).

\section{Overview Of circRNA Profiles}

Ten samples from five PHN patients were subjected to circRNA microarray. The Arraystar Human CircRNA Array v.2.0 detected 12,257 circRNAs (Supplementary material 4). Hierarchical clustering and scatter plot visualization showed the circRNAs expression levels were different (Figure 4), 
although only the hsa_circRNA_405463 show differential expression fold change $\geq 2.0$ between the PHN and control group. There were 23 circRNAs were up-regulated and 8 were down-regulated in PHN skin (fold change $\geq 1.5$ ), and if the threshold was set as 1.2 , up to 260 circRNAs were up-regulated and 154 were down-regulated in PHN skin (fold change $\geq 1.2$, Supplementary material 5). The top 10 up- and downregulated circRNAs are listed in Table 3.

\section{Discussion}

Skin is the lesion site and the location where hyperalgesia and allodynia appear in PHN patients. For the first time, the expression profiles of miRNAs and circRNAs in the PHN skin were detected with microarrays. Comprehensive expression changes of transcripts, especially miRNAs, were found between PHN and normal skin. This indicates that PHN skin and these differentially expressed molecules can be targets for PHN treatment.

miRNAs can regulate biological processes by inhibiting the function of their target genes. In this study, as many as 317 miRNAs were found differentially expressed in PHN skin, and 13 of them showed expression fold change $>10$, indicating that these differential miRNAs may influence their target genes' expression as well as PHN pathologies.
To evaluate functions of these miRNAs, their target mRNAs were predicted and KEGG pathway results indicated that they may influence the PHN pathology via AMPK, MAPK, ErbB, FoxO pathway, etc. It has been extensively reported that $\mathrm{AMPK},{ }^{31,32} \mathrm{MAPK}^{13,33,34}$ and $\mathrm{ErbB}^{35,36}$ pathway involved in chronic and neuropathic pain in animal studies. Although FoxO's function has not been confirmed in neuropathic pain model, bioinformatics predicted that FoxO pathway in the dorsal spinal $\operatorname{cord}^{27}$ or dorsal root ganglion ${ }^{37}$ was related to neuropathic pain.

It seems feasible and effective to interfere with the expression of miRNAs in skin. Animal studies suggested that interfering with the miRNA expression in the skin affects the prognosis of diseases. For example, interfering NR1 subunit of the NMDA receptor by intradermally injecting lentiviral vector-encoded miRNA-based shRNA reduced inflammatory pain in rats. ${ }^{38}$ Intradermally injecting miR129 and/or miR-335 agomirs into the wound edges accelerated wound healing in rats with diabetes. ${ }^{39}$ Some miRNAs have been intradermally given and tested in clinical trials. ${ }^{40}$

Exploration of circRNAs' roles in pain situation is still in infancy. Only a few studies focused on the expression change and mechanism of circRNA in pain situation. ${ }^{24-27}$ This study added evidence and suggested that PHN

Table 3 Differential circRNAs Between PHN Skin And Mirror Skin $(n=5)$

\begin{tabular}{|c|c|c|c|c|}
\hline CircRNA Name & Fold Change & Intensity (PHN) & Intensity (Control) & P-value \\
\hline \multicolumn{5}{|l|}{ Top 10 up-regulated in PHN } \\
\hline hsa_circRNA_101797 & 1.9 & 136.9 & 57.9 & 0.018 \\
\hline hsa_circRNA_0588I9 & 1.6 & 216.1 & 109.5 & 0.045 \\
\hline hsa_circRNA_063013 & 1.6 & 114.1 & 66.4 & 0.010 \\
\hline hsa_circRNA_404988 & 1.6 & 60.3 & 40.5 & 0.026 \\
\hline hsa_circRNA_407I76 & 1.6 & 317.5 & 158.4 & 0.009 \\
\hline hsa_circRNA_406848 & 1.5 & 70.1 & 42.8 & 0.040 \\
\hline hsa_circRNA_001379 & 1.5 & 536.7 & 271.5 & 0.008 \\
\hline hsa_circRNA_406106 & 1.5 & 213.7 & 116.3 & 0.036 \\
\hline hsa_circRNA_404432 & 1.5 & 392.4 & 209.9 & 0.003 \\
\hline hsa_circRNA_102538 & 1.5 & 183.1 & 106.7 & 0.014 \\
\hline \multicolumn{5}{|l|}{ Top 10 down-regulated in PHN } \\
\hline hsa_circRNA_405463 & -2.0 & 1809.7 & 2057.5 & 0.007 \\
\hline hsa_circRNA_077409 & -1.8 & 128.2 & 190.6 & 0.037 \\
\hline hsa_circRNA_0II 7772 & -1.8 & 82.6 & 122.9 & 0.029 \\
\hline hsa_circRNA_101731 & -1.8 & 160.5 & 235.2 & 0.007 \\
\hline hsa_circRNA_104137 & -1.8 & 1126.4 & 1502.8 & 0.002 \\
\hline hsa_circRNA_104664 & -1.7 & 263.1 & 347.6 & 0.026 \\
\hline hsa_circRNA_025988 & -1.7 & 51.5 & 78.2 & 0.004 \\
\hline hsa_circRNA_I01130 & -1.6 & 161.6 & 204.4 & 0.029 \\
\hline hsa_circRNA_I0134I & -1.6 & 101.9 & 142.1 & 0.002 \\
\hline hsa_circRNA_026457 & -1.6 & 3393.7 & 3519.8 & 0.022 \\
\hline
\end{tabular}


induced expression changes of circRNA in peripheral tissue. Further studies with gain- and/or loss-of-function experiments of these miRNAs and circRNAs will help understand their functions in PHN situation.

It is hard to tell whether the differential miRNAs and circRNAs are from the nerve endings of DRG neurons or cells that make up the skin. It is also not certain that these transcripts are present in cells because neurons and other cells may release miRNAs ${ }^{41}$ and circRNAs ${ }^{42}$ to the extracellular space via extracellular vesicles, such as exosomes. In situ hybridization experiments may help explain this question. Nevertheless, interventions targeting these differential miRNAs or circRNAs at the affected skin may help to alleviate PHN.

\section{Conclusions}

MiRNAs and circRNAs differentially expressed in the skin of PHN patients. Target genes of differential miRNAs enriched into pathways related to pathological pain regulation. These differential miRNAs and circRNAs as well as these predicted pathways could be potential targets to treat PHN.

\section{Acknowledgments}

The study was supported by a scientific research project from The Science and Technology Department of Guizhou Province (LH[2015]7554), the Innovative Training Program ([2015] 3109) of Zunyi Medical University and the Excellent Young Talents Project of Zunyi Medical University (18zy-004). This work was also supported by a PhD Startup Fund ([2017]21) of the Affiliated Hospital of Zunyi Medical University.

\section{Disclosure}

The authors report no conflicts of interest in this work.

\section{References}

1. Rowbotham MC, Davies PS, Fields HL. Topical lidocaine gel relieves postherpetic neuralgia. Ann Neurol. 1995;37(2):246-253. doi:10.1002/ ana.410370216

2. Pickering G, Gavazzi G, Gaillat J, Paccalin M, Bloch K, Bouhassira D. Is herpes zoster an additional complication in old age alongside comorbidity and multiple medications? Results of the post hoc analysis of the 12month longitudinal prospective observational ARIZONA cohort study. BMJ Open. 2016;6(2):e009689. doi:10.1136/bmjopen-2015-009689

3. Devor M. Rethinking the causes of pain in herpes zoster and postherpetic neuralgia: the ectopic pacemaker hypothesis. Pain Rep. 2018;3(6):e702. doi:10.1097/PR9.0000000000000702

4. Oaklander AL. Mechanisms of pain and itch caused by herpes zoster (shingles). J Pain. 2008;9(1 Suppl 1):S10-S18. doi:10.1016/ j.jpain.2007.10.003

5. Philip A, Thakur R. Post herpetic neuralgia. J Palliat Med. 2011;14 (6):765-773. doi:10.1089/jpm.2011.9685
6. Cao S, Li Y, Deng W, et al. Local brain activity differences between herpes zoster and postherpetic neuralgia patients: a resting-state functional MRI study. Pain Physician. 2017;20(5):E687-Ee699.

7. Cao S, Qin B, Zhang Y, et al. Herpes zoster chronification to postherpetic neuralgia induces brain activity and grey matter volume change. Am J Transl Res. 2018;10(1):184-199.

8. Cao S, Song G, Zhang Y, et al. Abnormal local brain activity beyond the pain matrix in postherpetic neuralgia patients: a resting-state functional MRI study. Pain Physician. 2017;20(2):E303-E314.

9. Baron R, Binder A, Wasner G. Neuropathic pain: diagnosis, pathophysiological mechanisms, and treatment. Lancet Neurol. 2010;9 (8):807-819. doi:10.1016/S1474-4422(10)70143-5

10. Andersen HH, Duroux M, Gazerani P. MicroRNAs as modulators and biomarkers of inflammatory and neuropathic pain conditions. Neurobiol Dis. 2014;71(11):159-168. doi:10.1016/j.nbd.2014. 08.003

11. Jiangpan P, Qingsheng M, Zhiwen Y, Tao Z. Emerging role of microRNA in neuropathic pain. Curr Drug Metab. 2016;17(4):336-344.

12. Fernandes V, Sharma D, Vaidya S, et al. Cellular and molecular mechanisms driving neuropathic pain: recent advancements and challenges. Expert Opin Ther Targets. 2018;22(2):131-142. doi:10.1080/ 14728222.2018.1420781

13. Inoue K, Tsuda M. Microglia in neuropathic pain: cellular and molecular mechanisms and therapeutic potential. Nat Rev Neurosci. 2018;19(3):138-152. doi:10.1038/nrn.2018.2

14. Huang Y, Li X, Tao G, Zhu T, Lin J. Comparing serum microRNA levels of acute herpes zoster patients with those of postherpetic neuralgia patients. Medicine (Baltimore). 2017;96(8):e5997. doi:10.1097/MD.0000000000005997

15. Leinders M, Uceyler N, Thomann A, Sommer C. Aberrant microRNA expression in patients with painful peripheral neuropathies. J Neurol Sci. 2017;380:242-249. doi:10.1016/j.jns.2017.07.041

16. Leinders M, Uceyler N, Pritchard RA, Sommer C, Sorkin LS. Increased miR-132-3p expression is associated with chronic neuropathic pain. Exp Neurol. 2016;283(Pt A):276-286. doi:10.1016/j. expneurol.2016.06.025

17. Leinders M, Doppler K, Klein T, et al. Increased cutaneous miR-let$7 \mathrm{~d}$ expression correlates with small nerve fiber pathology in patients with fibromyalgia syndrome. Pain. 2016;157(11):2493-2503. doi:10.1097/j.pain.0000000000000668

18. Wilusz JE, Sharp PA. A circuitous route to noncoding RNA. Science. 2013;340(6131):440-441. doi:10.1126/science. 1238522

19. Hansen TB, Jensen TI, Clausen BH, et al. Natural RNA circles function as efficient microRNA sponges. Nature. 2013;495 (7441):384-388. doi:10.1038/nature11993

20. Zheng Q, Bao C, Guo W, et al. Circular RNA profiling reveals an abundant circHIPK3 that regulates cell growth by sponging multiple miRNAs. Nat Commun. 2016;7(11215). doi:10.1038/ncomms11215

21. Thomson DW, Dinger ME. Endogenous microRNA sponges: evidence and controversy. Nat Rev Genet. 2016;17(5):272. doi:10.1038/nrg.2016.20

22. Memczak S, Jens M, Elefsinioti A, et al. Circular RNAs are a large class of animal RNAs with regulatory potency. Nature. 2013;495 (7441):333-338. doi:10.1038/nature11928

23. Wang Y, Yu X, Luo S, Han H. Comprehensive circular RNA profiling reveals that circular RNA100783 is involved in chronic CD28-associated CD8(+)T cell ageing. Immun Ageing. 2015;12(1):1-10. doi:10.1186/s12979-015-0028-X

24. Wang L, Luo T, Bao $\mathrm{Z}, \mathrm{Li} \mathrm{Y}, \mathrm{Bu}$ W. Intrathecal circHIPK3 shRNA alleviates neuropathic pain in diabetic rats. Biochem Biophys Res Commun. 2018;505(3):644-650. doi:10.1016/j. bbrc.2018.09.158

25. Zhou J, Xiong Q, Chen H, Yang C, Fan Y. Identification of the spinal expression profile of non-coding RNAs involved in neuropathic pain following spared nerve injury by sequence analysis. Front Mol Neurosci. 2017;10:91. doi:10.3389/fnmol.2017.00091 
26. Chu C, Chunshuai W, Jiajia C, et al. Transcriptional information revealed differentially expressed circular RNAs in facet joint osteoarthritis. Biochem Biophys Res Commun. 2018;497(2):790-796. doi:10.1016/j.bbrc.2018.02.157

27. Cao S, Deng W, Li Y, et al. Chronic constriction injury of sciatic nerve changes circular RNA expression in rat spinal dorsal horn. $J$ Pain Res. 2017;10:1687-1696. doi:10.2147/JPR.S139592

28. Cao S, Liu Y, Sun W, et al. Genome-wide expression profiling of anoxia/reoxygenation in rat cardiomyocytes uncovers the role of MitoKATP in energy homeostasis. Oxid Med Cell Longev. 2015;2015:756576. doi:10.1155/2015/659750

29. Su H, Lin F, Xia D, Shen L. Profiling and bioinformatics analyses reveal differential circular RNA expression in radioresistant esophageal cancer cells. J Transl Med. 2016;14(1):225. doi:10.1186/s12967-016-0867-z

30. Enright AJ, John B, Gaul U, Tuschl T, Sander C, Marks DS. MicroRNA targets in Drosophila. Genome Biol. 2003;5(1):R1. doi:10.1186/gb-2003-5-1-r1

31. Xiang $\mathrm{HC}$, Lin $\mathrm{LX}, \mathrm{Hu} \mathrm{XF}$, et al. AMPK activation attenuates inflammatory pain through inhibiting NF-kappaB activation and IL1beta expression. J Neuroinflamm. 2019;16(1):34.

32. Wang S, Kobayashi K, Kogure Y, et al. Negative regulation of TRPA1 by AMPK in primary sensory neurons as a potential mechanism of painful diabetic neuropathy. Diabetes. 2018;67(1):98-109. doi: $10.2337 / \mathrm{db} 17-0503$

33. Lin X, Wang M, Zhang J, Xu R. p38 MAPK: a potential target of chronic pain. Curr Med Chem. 2014;21(38):4405-4418. doi:10.2174/ 0929867321666140915143040

34. Huang CT, Chen SH, Lin SC, Chen WT, Lue JH, Tsai YJ. Erythropoietin reduces nerve demyelination, neuropathic pain behavior and microglial MAPKs activation through erythropoietin receptors on Schwann cells in a rat model of peripheral neuropathy. Glia. 2018;66(11):2299-2315. doi:10.1002/glia.23461
35. Dai DW, Xu Z, Chen X, et al. Distinct roles of neuregulin in different models of neuropathic pain. Neurol Sci. 2014;35(4):531-536. doi:10.1007/s10072-013-1537-z

36. Calvo M, Zhu N, Grist J, Ma Z, Loeb JA, Bennett DL. Following nerve injury neuregulin-1 drives microglial proliferation and neuropathic pain via the MEK/ERK pathway. Glia. 2011;59(4):554-568. doi:10.1002/glia.21124

37. Chen CJ, Liu DZ, Yao WF, et al. Identification of key genes and pathways associated with neuropathic pain in uninjured dorsal root ganglion by using bioinformatic analysis. J Pain Res. 2017;10:26652674. doi:10.2147/JPR.S143431

38. Liu CC, Cheng JT, Hung KC, Chia YY, Tan PH. Lentiviral vectorencoded microRNA-based shRNA-mediated gene knockdown of $\mathrm{N}$-methyl-D-aspartate receptors in skin reduces pain. Brain Behav. 2017;7(1):e00587. doi:10.1002/brb3.587

39. Wang W, Yang C, Wang XY, et al. MicroRNA-129 and -335 promote diabetic wound healing by inhibiting Sp1-mediated MMP-9 expression. Diabetes. 2018;67(8):1627-1638.

40. Singhvi G, Manchanda P, Krishna Rapalli V, Kumar Dubey S, Gupta G, Dua K. MicroRNAs as biological regulators in skin disorders. Biomed Pharmacother. 2018;108:996-1004. doi:10.1016/j.biopha. 2018.09.090

41. Xu B, Zhang Y, Du XF, et al. Neurons secrete miR-132-containing exosomes to regulate brain vascular integrity. Cell Res. 2017;27 (7):882-897. doi:10.1038/cr.2017.62

42. Kim KM, Abdelmohsen K, Mustapic M, Kapogiannis D, Gorospe M. RNA in extracellular vesicles. Wiley Interdiscip Rev. 2017;8(4): e1413. doi:10.1002/wrna.2017.8.issue-4
Journal of Pain Research

\section{Publish your work in this journal}

The Journal of Pain Research is an international, peer reviewed, open access, online journal that welcomes laboratory and clinical findings in the fields of pain research and the prevention and management of pain. Original research, reviews, symposium reports, hypothesis formation and commentaries are all considered for publication. The manuscript management system is completely online and includes a very quick and fair peer-review system, which is all easy to use. Visit http:// www.dovepress.com/testimonials.php to read real quotes from published authors. 